\title{
DEVELOPING A CAMPUS WIDE BUILDING INFORMATION SYSTEM BASED ON OPEN STANDARDS
}

\author{
Andrea Buda ${ }^{1}$, Tuomas Kinnunen ${ }^{2}$, Bhargav Dave ${ }^{3}$ and Kary Främling ${ }^{4}$
}

\begin{abstract}
University campuses can significantly benefit from IoT technologies, especially from operational efficiencies and user experience perspective. Traditionally, such systems have been limited to lab based environments, where involvement of end-users is limited and the results may not reflect reality. To build IoT systems for real-world that are reliable and relevant, it is important to build experiments in real-world conditions and involve end-users. From technological perspective, there is a need for convergence of diverse fields ranging from Building Information Systems and Building Services to Building Automation Systems, IoT devices and finally the campus services that include academic and research activities.

This paper outlines the efforts to develop a campus wide web based system called Otaniemi3D that provides information about energy usage, occupancy and user comfort by integrating Building Information Models and IoT devices through open messaging standards (O-MI and O-DF) and IFC models. The paper describes the design criteria and the system architecture and the workflow to generate the information needed to develop such a system.
\end{abstract}

Keywords: Internet of Things, BIM, SmartCampus, Open Standards.

\section{INTRODUCTION}

Campuses - University, School or Organisational, are a highly dynamic environment represented by a diverse range of stakeholders, where space, time and resource management are key challenges. IoT technologies can play a significant role in smooth operations, monitoring, and control of such facilities. However vertical silos and proprietary (non-open) technologies are a barrier for innovative cross-domain and crossplatform implementations, and hinder wider proliferation and research of such initiatives.

Recent advances in technologies such as IoT (Internet of Things), wireless sensors, data processing and analysis and Building Information Modelling (BIM) have the potential to transform how we interact with the built environment and improve the experience for end users and service providers (Nati et al. 2013). The IoT devices and sensors are increasingly being deployed in the built environment and industrial applications. The number of connected devices have already overtaken the connected human beings and are estimated to be around 9 billion. The sensor nodes are being deployed in various application areas such as industrial, transportation, building automation, automotive and

1 Doctoral Student, Department of Computer Science, Aalto University, Espoo, Finland, andrea.buda@aalto.fi

2 Master Student, Department of Computer Science, Aalto University, Espoo, Finland, tuomas.kinnunen@aalto.fi

3 Senior Researcher, Department of Computer Science, Aalto University, Espoo, Finland, bhargav.dave@aalto.fi

4 Professor of Practice, Department of Computer Science, Aalto University, Espoo, Finland, kary.framling@aalto.fi 
retail. The number of sensor installation is increasing at an exponential rate and some estimates suggest that there will be around 50 billion connected devices by 2020 (Gubbi et al. 2013).

The field of Building Information Modelling (BIM) is rapidly expanding as an information delivery and management platform for the built environment. BIM models are used across the entire project lifecycle including design and construction to operations and maintenance (Eastman 2011). With the emerging popularity of the BIM platforms, there is an opportunity to leverage this technology so that it can be used to build open platforms that synchronise with diverse information sources such as wireless sensors and building automation systems. With the built environment being the largest application area for IoT devices, the research that integrates the information standards that represent the building and the infrastructure with open IoT standards is an important research domain.

University Campuses provide excellent opportunities for active participation of researchers and students to develop innovative solutions and use cases for a developing technological area such as IoT (HUANG et al. 2012; Adamkó, Kãdek and Kósa 2014; Elger and Russell 2003). However, such opportunities are also somewhat restricted due to point wise implementation of proprietary technologies that do not provide open interfaces. Also, similar past projects have mainly been conducted in a lab based environment that provide limited potential to engage with real-life users (Nati et al. 2013).

The paper begins with design rationale that explains the main factors that influenced the design of the system. A description of system architecture follows that explains the main components of the system. Subsequently, the proof of concept implementation Otaniemi3D is described in detail followed by discussion and conclusion.

\section{DESign RATIONALE}

The aim of this research is to connect spaces/building with the data they generate through IoT devices with people living those spaces (students and researchers) as shown in Figure 1. The reason behind this aim is to achieve the following objectives:

Campus-wide system including real spaces and users: IoT-research is often confined in labs and small testbeds, acting more as proof of concepts and demos than reallife systems. Deploying a campus wide installation provides a wide range of challenges (scalability, security, privacy, purpose, beyond flashy demos, etc.), which are significant research and learning opportunities.

Multidisciplinary Research and Learning Platform: As mentioned in the previous point, an important aspect of the system is to provide "challenges" and "opportunities" for learning and research for students and researchers. From this context, the platform developed must be open and accessible as possible. It is very important to reach research groups working in areas other than IoT and BIM in order to realize the full potential of this kind of installation. 


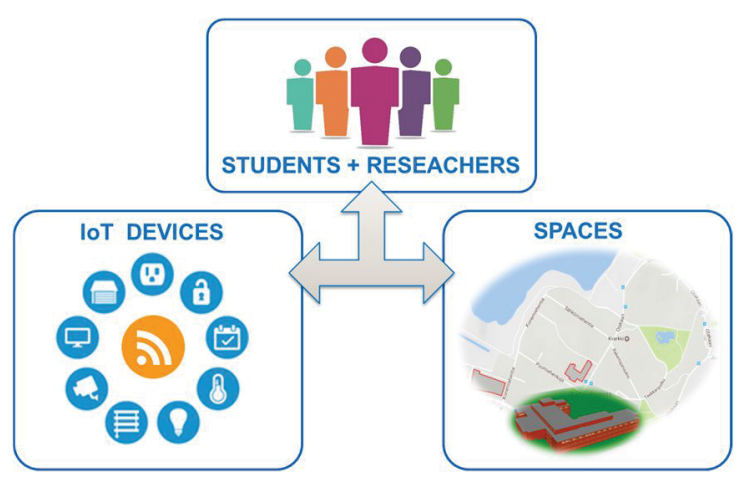

Figure 1: SmartCampus project aim

Besides the general aim and objectives, there is a set of more concrete considerations and goals, based on previous research conducted by the authors of this paper, which represent the foundation of this research:

1. Develop an end-to-end open source, secure IoT stack, from devices' firmware to web services.

2. Provide programmatic data-access using standardized Web-API.

3. Connect IoT data with the context and spaces in which it has been generated.

Humans have significant cognitive abilities to understand/interpret 3D spaces as compared with two dimensional representations of spatial information. This knowledge can be useful when analysing data: for example a temperature sensor positioned close to a window might be the reason why the recorded value is an outlier in the considered data set. In addition, there are important usability considerations behind this objective. Plaindata, in a database or flat files is often hard to understand (what it is about, which sensor, from where it comes from etc.), as it often lacks the necessary documentation to be properly used. Leveraging again on the strong human understanding of 3D spaces, the "motto" of the GUI (graphical user interface) of the system is "What you see physically is what get digitally". The GUI is a 1:1 representation of the real-world. "Whenever I see a sensor in the real world, I am able to navigate to same digital space and retrieve the associated data." This concept is further explained in section 3.3.

\section{SYSTEM ARCHITECTURE}

The architecture of the developed system can be broken down in 3 main components classes and related subcomponents (see Figure 2):

1. IoT Devices: Their primary function is to sense and act upon the environment in which they have been installed. The following section will briefly describe the firmware implementing reading sensors and controlling actuators, and the communication protocol with the backend server.

2. Backend Server: A collection of loosely coupled services communicating with each other's following the SOA (Service Oriented Architecture) design style. The data and functionality of each component is exposed to multiple front-ends via the standardized interface O-MI and O-DF (Open Messaging Interface and Open Data Format). The following section will describe in greater detail the BIM service and the IoT service.

3. Front end(s): The primary function of the front end is to manage the interaction with the target users of the system (students and researchers). In practice the front 
end can be implemented as websites or smartphone apps. They consume data from the backend, transforming into a human friendly form. In addition some of these front-ends is used to collect user inputs and data and feeding back to the backend system. So far only one front end has been developed, which will be described in the following section. However, one of the main goal of the entire smart campus project is to spark the development of a multitude of apps, leveraging on the competencies and interests of various research groups. In the vision, providing programmatic open-access to data is paramount for realizing the true potential of this type of systems. Section 3.3 will present one of these front-ends.

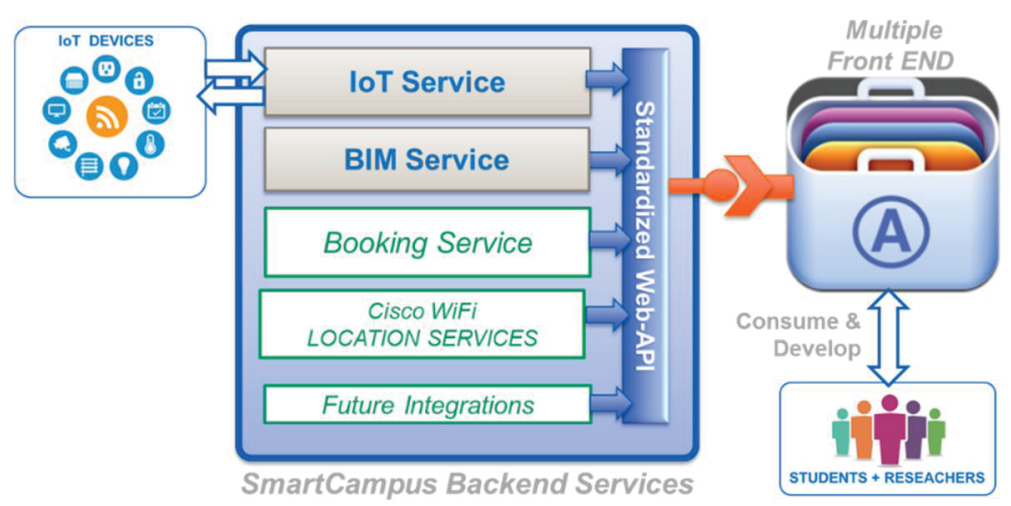

Figure 2: System Architecture

\section{OtANIEMI3D - PROOF OF CONCEPT}

This section describes the main components behind the development of Otaniemi3D - an open campus wide platform that integrates Building Information using IFC with wireless sensor nodes through Open APIs.

\subsection{Standardized Web-API}

The cornerstone of the entire Smart Campus backend is harmonize the publishing and consumption of data though a standardized Web-API. The selected standard are the Open Messaging Interface (O-MI) (Open Group IoT Standard) and the Open Data Format (ODF) (Open Group IoT Standard) published by the Open Group (TOG), IoT work group in 2014. The TOG-IoT work group has an ambitious vision: "Whereas the Web uses the HTTP protocol for transmitting HTML-formatted information which are rendered in the browser for human consumption, the IoT will use O-MI for transmitting O-DF payloads which will be mainly consumed by information systems."

A detailed explanation of these standards is outside the scope of this paper, nevertheless Figure 3 depicts the core operation supported by O-MI and its transported payload. The key characteristics of these standards are:

1. Transport agnostic: O-MI runs on top of existing transport level protocol. In general HTTP and WebSockets are the preferred protocols.

2. Publication and discovery of data sources and semantic meta-data: The data and methods available provided by a given node can be discovered using the ReadAll operation. In addition O-DF tags can be semantically enriched using RDFa and LinkedData vocabularies. 
3. Payload agnostic: Even though the preferred payload is O-DF (XML formatted), within specific O-DF tags any payload could be transported (CSV, HTML, proprietary file formats), or even binary file formats converted using Base64 binary-to-text encoding.

4. Support for Subscription: The possibility to create ad hoc, time-limited information flows by specifying for how long (TTL) and at which sampling rate (INTERVAL) I need to receive the data, is the cornerstone of O-MI and what makes it particularly suited for IoT and Industrial Internet applications.
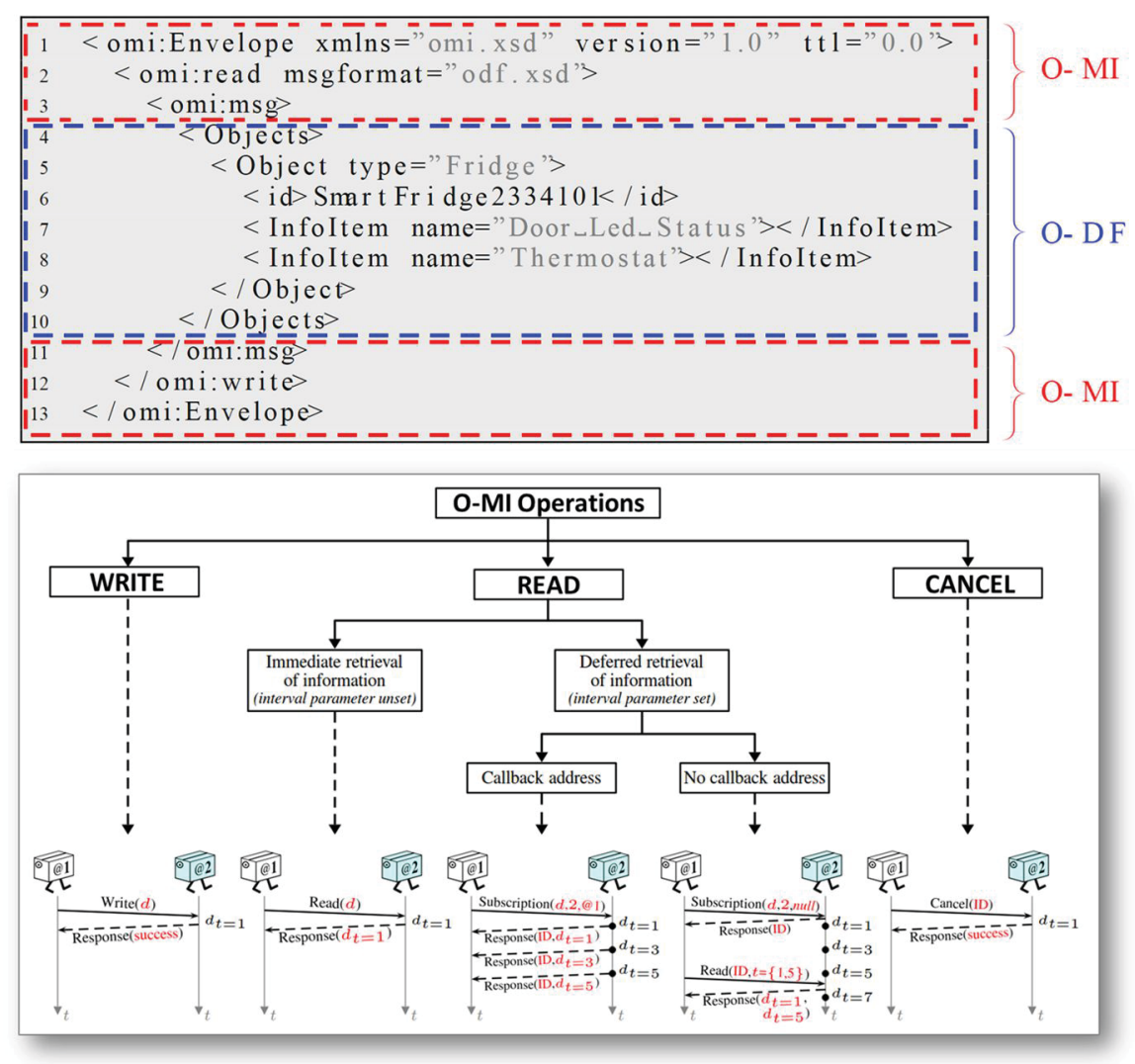

Figure 3: Top: O-MI and O-DF payload. Bottom: O-MI Main Operation

\subsection{IoT Devices and Service}

The IoT-Service has been implemented using the open source reference implementation of the O-MI and O-DF standards, developed at Aalto University (available online at https://github.com/AaltoAsia/O-MI). This particular implementation is an agent-based system: Agents are simple programs, which in this instance are in charge of encapsulating the complexity of the lower level communication with IoT-devices and the interaction with other services of the SmartCampus backend. The data collected from the devices is stored in Warp10 (http://www.warp10.io) an open source Geo Time Series ${ }^{\circledR}$ (GTS) database.

\subsection{BIM Service and Front End}

This service is essentially used to manage the relationship between spaces/buildings (described using IFC) and IoT-data, in particular the translation of static IFC file into interactive web document. Figure 5 describes the overall workflow and some important 
considerations regarding how the BIM model must be built. The translation toolchain adopted, required some custom programming for retaining the association between the IfcSensorType and the final output web formats. The process described is very similar to one adopted by BIMServer (http://bimserver.org/). The integration with the BIMServer is part of ongoing work and will be available in future integration of the SmartCampus backend.

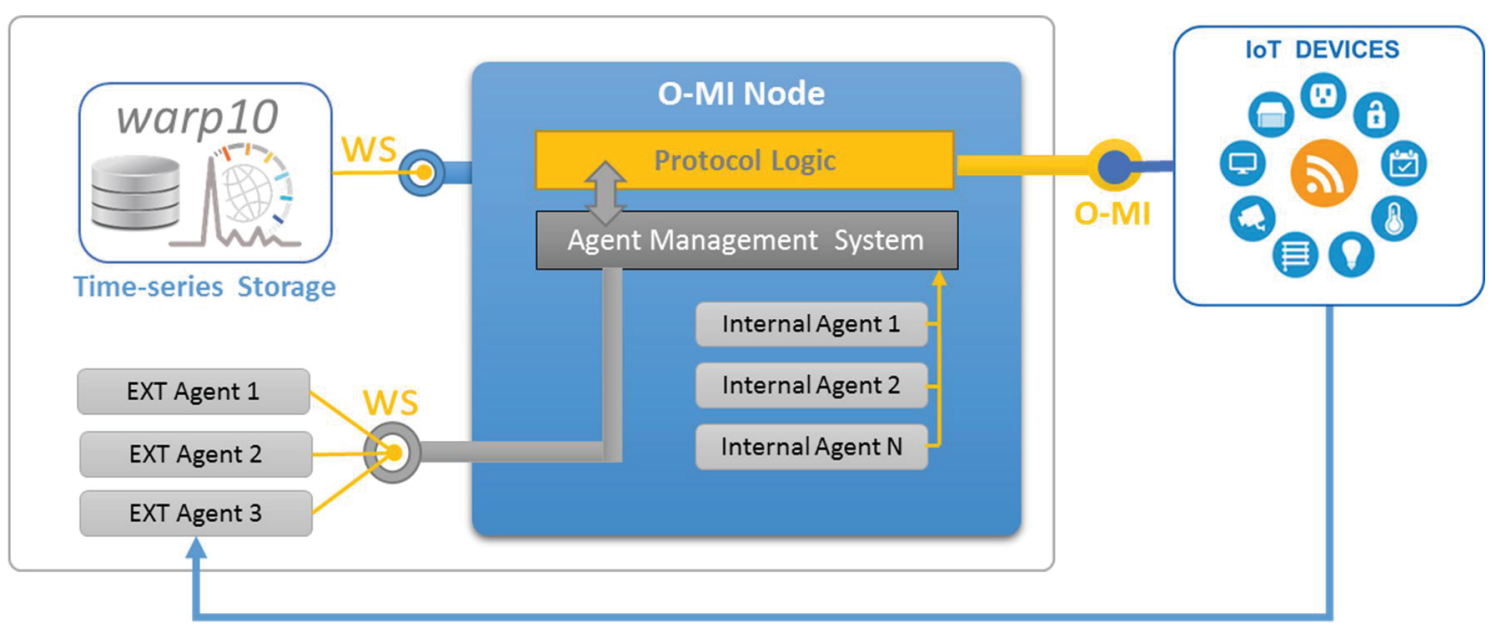

Figure 4: IoT - Service Main Components. WS stands for WebSocket.

Finally, Figure 6 depicts the main pages of the front-end of the system. The webapp (available at https://otaniemi3d.cs.hut.fi) has been called Otaniemi3D, where Otaniemi is the name of the Aalto University Campus and 3D stands for the dimension in which the BIM and IoT data is presented (1D) Data-Analytics Page (2D) Floor Plan and heatmaps page (3D) 3D Model and precise sensor location.

\subsection{Potential use cases}

With an open framework, the authors envisage that a variety of use cases can be developed to demonstrate the capability of the system. The following describes the use cases which can be supported by the GUI described above.

\subsubsection{Living Lab}

With sensors installed, integrated with spatial information and providing live data in a real-life setting through an open framework, researchers and students can use this to conduct analysis to support research projects, for example identifying user comfort patterns with changing sensor data.

\subsubsection{Intelligent Room booking system}

With the spatial information from IFC model, integration with room availability through open APIs, campus inhabitants (students, staff, researchers, etc.) can use the system to identify the best available rooms to match their need. With the room size, live sensor data and location, the users can be directed to the best suitable room that will also be energy efficient for the purpose.

\subsubsection{Facility management}

The IFC data provides accurate information about the building components and their design specifications, which along with the real-life sensor data can be used as a dashboard for facilities management. 


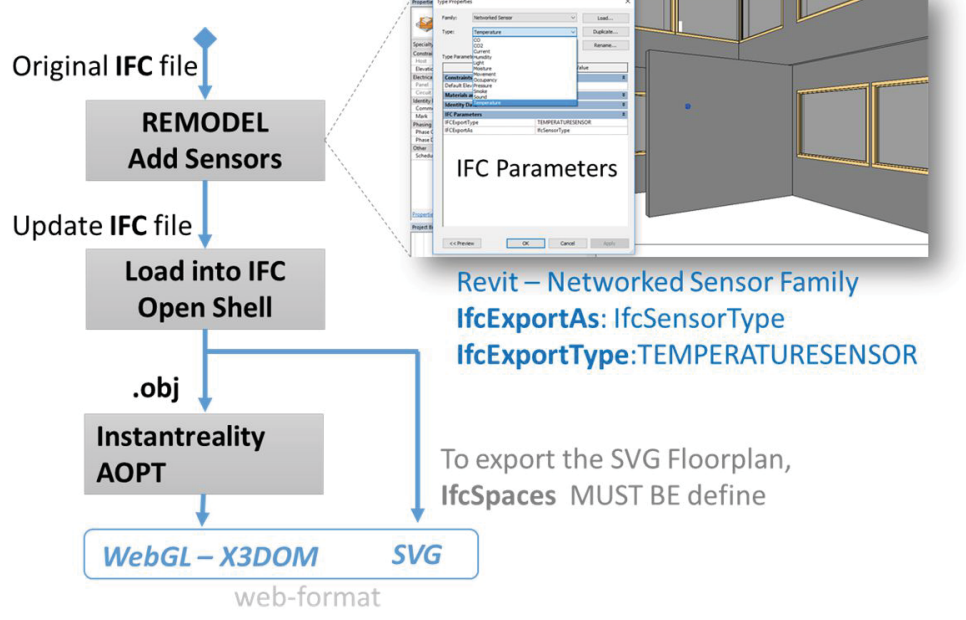

Figure 5: IoT - Service Main Components. WS stands for WebSocket

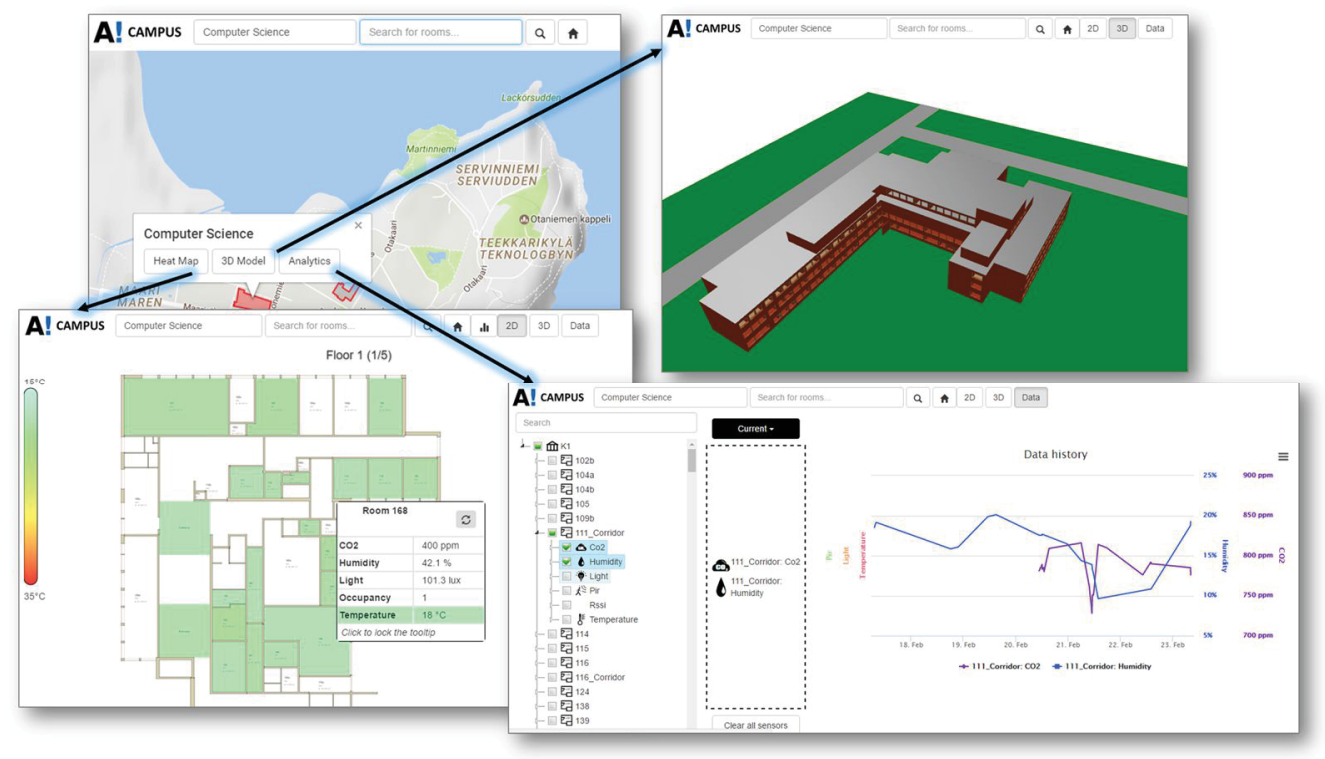

Figure 6: Otaniemi3D - SmartCampus FrontEnd

\section{CONCLUSIONS}

A large proportion of IoT devices are deployed in the built environment providing an opportunity to develop interfaces that allow user interaction through open, intuitive interfaces. This research shows that it is possible to engage wide range of stakeholders with IoT devices by integrating them with building information data. The communication takes place through Open-Messaging interfaces eliminating the need to depend on closed proprietary systems that hinder scalable deployment of such systems and in an intuitive 3D environment. There are several challenges that present themselves while developing such a platform and especially in implementation. Without standardised export guidelines for IFC files, and parsers to export this data for web, it is challenging to map sensor data to objects in IFC. Additionally, developing open yet secure interfaces at the campus level 
(with diverse user profiles) poses an additional challenge along with detection and prevention of rogue nodes. In future, researchers can develop more detailed implementations that address these challenges and address real-life requirements.

\section{REFERENCES}

Adamkó, A., T. Kãdek, and M. Kósa. (2014). Intelligent and Adaptive Services for a Smart Campus." In 2014 5th IEEE Conference on Cognitive Infocommunications (CogInfoCom), 505-9. doi:10.1109/CogInfoCom.2014.7020509.

Eastman, C. M. (2011). BIM Handbook: A Guide to Building Information Modeling for Owners, Managers, Designers, Engineers and Contractors. 2nd ed. Hoboken, NJ: Wiley.

Elger, D. and Russell, P. (2003). The Virtual Campus: A New Place for (Lifelong) Learning? Automation in Construction, Design e-ducation: Connecting the Real and the Virtual, 12 (6): 671-76. doi:10.1016/S0926-5805(03)00046-3.

Gubbi, J., Rajkumar B., Slaven M., and Marimuthu P. (2013). Internet of Things (IoT): A Vision, Architectural Elements, and Future Directions. Future Generation Computer Systems, Including Special sections: Cyber-enabled Distributed Computing for Ubiquitous Cloud and Network Services \& Cloud Computing and Scientific Applications - Big Data, Scalable Analytics, and Beyond, 29 (7): 1645-60. doi:10.1016/j.future.2013.01.010.

Huang, R., Zhang, J., Hu, Y. and Yang, J. (2012). Smart Campus: The Developing Trends of Digital Campus. Open Education Research 4: 4.

Nati, M., Gluhak, A., Abangar, H. and Headley, W. (2013). Smartcampus: A User-Centric Testbed for Internet of Things Experimentation. In Wireless Personal Multimedia Communications (WPMC), 2013 16th International Symposium on, 1-6. IEEE. http://ieeexplore.ieee.org/abstract/document/6618632/.

Open Data Format (O-DF), an Open Group Internet of Things (IoT) Standard, US ISBN 1937218-59-1, Oct. 2014, Available online at https://www2.opengroup.org/ogsys/catalog/C14A

Open Messaging Interface (O-MI), an Open Group Internet of Things (IoT) Standard, US ISBN 1-937218-60-7, Oct. 2014, Available online at https://www2.opengroup.org/ogsys/catalog/C14B 